\title{
The Asymptotic Eigenvalues of First-Order Spectral Differentiation Matrices
}

\author{
Jue Wang1, Fabian Waleffe ${ }^{2}$ \\ ${ }^{1}$ Department of Mathematics, Union College, Schenectady, USA \\ ${ }^{2}$ Department of Mathematics, University of Wisconsin-Madison, Madison, USA \\ Email: wangj@union.edu
}

Received January 2014

\section{Abstract}

We complete and extend the asymptotic analysis of the spectrum of Jacobi Tau approximations that were first considered by Dubiner. The asymptotic formulas for Jacobi polynomials $P_{N}^{(\alpha, \beta)}, \alpha, \beta>-1$ are derived and confirmed by numerical approximations. More accurate results for the slowest decaying mode are obtained. We explain where the large negative eigenvalues come from. Furthermore, we show that a large negative eigenvalue of order $N^{2}$ appears for $-1<\alpha<0$; there are no large negative eigenvalues for collocations at Gauss-Lobatto points. The asymptotic results indicate unstable eigenvalues for $\alpha>1$. The eigenvalues for Legendre polynomials are directly related to the roots of the spherical Bessel and Hankel functions that are involved in solving Helmholtz equation inspherical coordinates.

\section{Keywords}

Asymptotic Analysis, Spectral Approximations, Jacobi Polynomials, Collocation, Eigenvalues

\section{Introduction}

Pseudospectral methods were developed to solve differential equations, where derivatives are computed numerically by multiplying a spectral differentiation matrix [1]. Compared to finite difference methods that use local information, pseudospectral methods are global, and have exponential rate of convergence and low dissipation and dispersion errors. However in boundary value problems, they are often subject to stability restrictions [2]. If the grid is not periodic, the spectral differentiation matrices are typically nonnormal [3], and the nonnormality may have a big effect on the numerical stability and behavior of the methods. On a grid of size $N$, pseudos- pectral methods require a time step restriction of $O\left(N^{-2}\right)$ [4] for hyperbolic and $O\left(N^{-4}\right)$ [5] for parabolic problems.

Dubiner [6] carried an asymptotic analysis for theone-dimensional wave equation. He pointed out the $O(N)$ boundedness of the eigenvalues of the spectral differentiation matrix with collocation at Legendre points. It was also proposed in [7] that the $O\left(N^{2}\right)$ eigenvalues could be shrunk to $O(N)$ byreplacing Chebyshev Tau method with 
Legendre Tau method. That would mean a time step size increase from $O\left(N^{-2}\right)$ to $O\left(N^{-1}\right)$. However, the eigenvalues of Chebyshev and Legend respectral differentiation matrices are extremely sensitive to rounding errors and other perturbations [4]. On agrid of size $N$, machine rounding could lead to errors of size $O\left(N^{2}\right)$. Thus the Legendre Tau method which has an $O\left(N^{-1}\right)$ time step restriction in theory, is subject to an $O\left(N^{-2}\right)$ restriction in practice.

The slowest decaying mode and largest wave numbers are often of interest. They affect stabilities and limit time step sizes of pseudospectral approximations. It is reported in [6] [8] that there exists a large negative eigenvalue of order $N^{2}$ for Chebyshev spectral differentiation matrix. Where does that eigenvalue come from? When does it appear? And how does it affect the time step size? In this paper, we will consider the first-order spectral differentiation matrix and examine the behavior of its eigenvalues asymptotically and numerically.

Let's consider the first-order eigenvalue problem

$$
\frac{d u}{d x}=\lambda u,-1<x<1, u(1)=0
$$

The spectrum of the differentiation operator is empty. However, the spectrum of the Tau approximations to the eigenvalue problem affects the stability of the associated wave equation $u_{t}=u_{x}$ on $(-1,1)$ with boundary condition $u(1, t)=0$, whose solution is a leftward translation at speed 1 [3]. Let $u_{N}(x)$ be a polynomial approximation of degree $N \cdot u_{N}(x)$ satisfies

$$
\lambda u_{N}-D u_{N}=P_{N}(x), u_{N}(1)=0
$$
where $D \equiv d / d x, P_{N}(x)$ is a polynomial of degree $N$. We can write $u_{N}(x)=-e^{\lambda x} \int_{a}^{x} e^{-\lambda s} P_{N}(s) d s$ for some arbi-
trary constant $a$. Integration by parts gives

$$
u_{N}(x)=\frac{1}{\lambda} \sum_{k=0}^{N} \frac{D^{k} P_{N}(x)}{\lambda^{k}}-\frac{e^{\lambda(x-a)}}{\lambda} \sum_{k=0}^{N} \frac{D^{k} P_{N}(a)}{\lambda^{k}} .
$$

The non-polynomial contribution (the second term) is eliminated by picking $a=-\infty$ if $\mathfrak{R} \lambda<0$, and $a=+\infty$ if $\Re \lambda>0$. We then obtain

$$
u_{N}(x)=\frac{1}{\lambda} \sum_{k=0}^{N} \frac{D^{k} P_{N}(x)}{\lambda^{k}}
$$

The boundary condition $u_{N}(1)=0$ implies

$$
\begin{gathered}
-\int_{-\infty}^{1} e^{\lambda(1-s)} P_{N}(s) d s=0, \Re \lambda<0 \\
\int_{1}^{\infty} e^{\lambda(1-s)} P_{N}(s) d s=0, \Re \lambda>0
\end{gathered}
$$

In the Tau method [1], the polynomial approximation $u_{N}(x)$ is determined from the boundary condition $u_{N}(1)=0$ and the requirement that $P_{N}(x)$ is orthogonal to all polynomials of degree $n-1$ with respect to the weight function $w(x) \geq 0$ in the interval $(-1,1)$. For Jacobi weight function $w_{(\alpha, \beta)}(x)=(1-x)^{\alpha}(1+x)^{\beta}$, $P_{N}(x)=P_{N}^{(\alpha, \beta)}(x)$, the Jacobi polynomial of degree $N$ with $\alpha, \beta>-1$.

Assume $\lambda \neq 0$, otherwise $\lambda=0$ corresponds to the trivial solution $u_{N}(x)=0$. From (3), the boundary condition $u_{N}(1)=0$ leads to the characteristic polynomial $\Phi_{N}(v)=\sum_{k=0}^{N^{N}} D^{k} P_{N}(1) v^{k}$, with $v=1 / \lambda$. It is proved [9] that the eigenvalues lie in the left half-plane for Jacobi polynomial $P_{N}^{(\alpha, \beta)}(x)$ if $-1<\alpha \leq 1$ and $\beta>-1$. The eigenvalues are computed numerically using the three term recurrence relation for corresponding Jacobi polynomials [10] (see Figure 1). We will show that the theorem is sharp by obtaining asymptotic results indicating unstable eigenvalues for $\alpha>1$.

In order to obtain the asymptotic behavior for large values of $\lambda$, we use the method of steepest descents to deform the integration paths to obtain the dominant contribution from saddle points. In general there are two saddle and two boundary points. The balance between the dominant saddle and boundary contributions leads to an asymptotic equation for $\lambda$. The two saddle points collide to form a third-order saddle when $\lambda / N= \pm i$; nearly merge when $\alpha=0$ and $|\lambda / N \pm i| \ll 1$; and are too close to the boundary points when $|\lambda|>O(N)$. These cases complicate the analysis. 


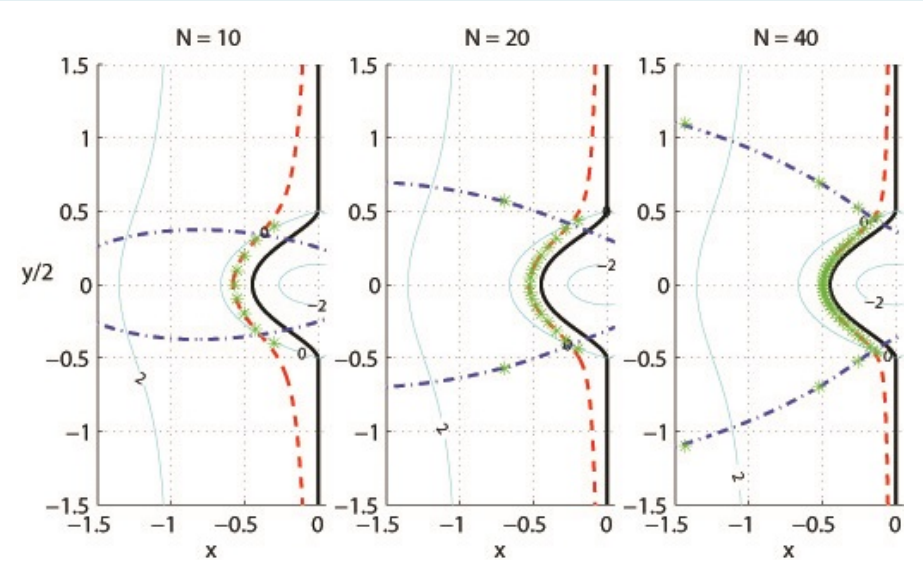

Figure 1. The limit curve and eigenvalue approximations for Chebyshev polynomials ( $\alpha=\beta=\frac{-1}{2}$ ). Thick solid: $F(\mu, \sigma=-1)=0$, dash: $F(\mu, \sigma=-1)=-\frac{3}{2} \frac{\ln N}{N}-O\left(\frac{1}{N}\right)$, dash-dot: $F(\mu, \sigma=1)=-\frac{3}{2} \frac{\ln N}{N}$ $-O\left(\frac{1}{N}\right)$, where $F(\mu, \sigma)=\Re\left\{\mu+\sigma \sqrt{1+\mu^{2}}+\ln \frac{1-\sigma \sqrt{1+\mu^{2}}}{\mu}\right\}$.

Thin solid: contours of $B(\mu)$. Take dash-dot branch for $B(\mu)>0$, dash for $B(\mu)<0$.

The paper is organized as follows. In Section 2, we present the asymptotic analysis and numerical results for Chebyshev polynomials. We generalize the results to Jacobi polynomials in Section 3, and derive the approximations of the slowest decaying mode and largest wave numbers. In Section 4, we show that the eigenvalues for Legendre polynomials are directly related to the roots of spherical Bessel and Hankel functions. The analysis and numerical results for collocation methods are explained in Section 5. Finally we conclude in Section 6.

\section{Chebyshev Polynomials}

We use Chebyshev polynomials $P_{N}(s) \equiv T_{N}(s)$ to illustrate the approach and derive the asymptotic formulas. They correspond to the class of Jacobi polynomials with $\alpha=\beta=-1 / 2$ and are especially relevant in practice. With a change of variable $s=\cos \phi, T_{N}(\cos \phi)=\cos N \phi$, and (4) becomes

$$
\int_{\pi+i \infty}^{0} e^{-\lambda \cos \phi} \cos N \phi(-\sin \phi) d \phi=0
$$

It suggests that $\lambda=N \mu$. Define $\rho(\phi)=-\mu \cos \phi+i \phi$, and $I$ twice the above integral,

$$
I=\int_{0}^{\pi+i \infty}+\int_{2 \pi}^{\pi-i \infty} e^{N \rho(\phi)} \sin \phi d \phi=I_{1}+I_{2}
$$

We will apply the method of steepest descent to deform the integration path without changing the value of the integral, so that it goes through the critical point (saddle point) $\phi_{*}$ in such a way that $\mathfrak{R} \rho\left(\phi_{*}\right)$ is maximum along the path, and $\mathfrak{R} \rho\left(\phi_{*}\right)$ decreases along either direction away from $\phi_{*}$ as rapidly as possible. As $N \rightarrow \infty$, the dominant contributions come from the saddle points and boundary points 0 and $2 \pi$.

\subsection{Saddle Contributions}

The saddle points of $\rho(\phi)$ satisfy the equation $\rho^{\prime}(\phi)=\mu \sin \sin \phi+i=0$, which implies $\sin \phi_{*}=-i / \mu$. The steepest-descent curves of $e^{N \rho(\phi)}$ vary as $\mu$ varies. We consider two cases: $\mathfrak{R} \mu \neq 0$ and $\mathfrak{R} \mu=0$.

\subsection{1. $\Re \mu \neq 0$}

There are two saddle points $\phi_{*}^{(\sigma)}, \sigma=\operatorname{sign} \Im \phi_{*}^{(\sigma)} \neq 0$, and $\rho^{\prime \prime}\left(\phi_{*}^{(\sigma)}\right) \neq 0$. They satisfy the relationship 


$$
\begin{array}{r}
\phi_{*}^{(-1)}=\pi-\phi_{*}^{(1)}, \mathfrak{R} \rho\left(\phi_{*}^{(-1)}\right)=-\mathfrak{R} \rho\left(\phi_{*}^{(1)}\right), \mathfrak{I} \rho\left(\phi_{*}^{(-1)}\right)=\pi-\mathfrak{I} \rho\left(\phi_{*}^{(1)}\right), \text { and } \\
\rho\left(\phi_{*}^{(\sigma)}\right)=-\mu \cos \phi_{*}^{(\sigma)}+i \phi_{*}^{(\sigma)}=\sigma \sqrt{1+\mu^{2}}+\ln \frac{1-\sigma \sqrt{1+\mu^{2}}}{\mu}
\end{array}
$$

The integration paths follow the constant-phase contours $\Im \rho(\phi)=\Im \rho\left(\phi_{*}^{(\sigma)}\right)$, i.e. the steepest-descent curves, from 0 to $\pi+i \infty$ for $I_{1}$ and from $2 \pi$ to $\pi-i \infty$ for $I_{2}$, passing through the saddle points $\phi_{*}^{(\sigma)}$. The steepest curves corresponding to the opposite signs of $\Im \mu$ are mirror images about $\mathfrak{R} \phi=\pi$ axis. Define

$$
B(\mu) \equiv \mathfrak{R} \rho\left(\phi_{*}^{(1)}\right)-\mathfrak{R} \rho\left(\phi_{*}^{(-1)}\right)=\mathfrak{R}\left\{2 \sqrt{1+\mu^{2}}+\ln \frac{1-\sqrt{1+\mu^{2}}}{1+\sqrt{1+\mu^{2}}}\right\}
$$

and redefine $\sigma$ to be $\sigma=\operatorname{sign} B(\mu)$. Then $I^{s}\left(\phi_{*}{ }^{\sigma}\right)$ dominates when $B(\mu) \neq 0$.

Following the standard saddle point approximation ([11] §7.3), we evaluate the integrals near the saddle points and obtain the dominant contribution

$$
I^{s}=I^{s}\left(\phi_{*}^{\sigma}\right) \sim N^{-1 / 2} e^{N \rho\left(\phi_{*}^{(\sigma)}\right)} e^{i \theta^{(\sigma)}} \sin \phi_{*}^{(\sigma)} \sqrt{\frac{2 \pi}{\left|\rho^{\prime \prime}\left(\phi_{*}^{(\sigma)}\right)\right|}}
$$

As $N \rightarrow \infty$ when $B(\mu) \neq 0$.

If $B(\mu)=0, I^{s}=I^{s}\left(\phi_{*}^{(1)}\right)+I^{s}\left(\phi_{*}^{(-1)}\right)$.

\subsection{2. $\mathfrak{R} \mu=0$}

Let $\mu=i b$. The two saddles points collide and form athird-order saddle point at $\mu= \pm i$.

1) $0<|b|<1$. In this case $B(\mu)<0$.

$\phi_{*}^{( \pm 1)}=\pi / 2 \pm i\left|\cosh ^{-1}(-1 / b)\right|$ when $-1<b<0,3 \pi / 2 \pm i\left|\cosh ^{-1}(1 / b)\right|$ when $0<b<1$.

$I^{s}=I^{s}\left(\phi_{*}^{(-1)}\right)$.

2) $|b|>1$. We have $B(\mu)=0$.

$\phi_{*}^{(1)}=\sin ^{-1}(-1 / b), \quad \phi_{*}^{(-1)}=\pi-\sin ^{-1}(-1 / b)$ when $b<-1 . \quad \phi_{*}^{(1)}=2 \pi+\sin ^{-1}(-1 / b)$,

$\phi_{*}^{(-1)}=\pi-\sin ^{-1}(-1 / b)$ when $b>1$.

$$
I^{s}=I^{s}\left(\phi_{*}^{(1)}\right) .
$$

3) $b= \pm 1 . \phi_{*}=\pi+b \pi / 2$ is a third-order saddle point. A similar saddle point approximation leads to $I^{s}\left(\phi_{*}\right) \sim N^{-\frac{1}{3}} e^{i N \phi_{*}}\left(-e^{i\left(\frac{\pi}{2}-\frac{b \pi}{3}\right)}+e^{-\frac{i \pi}{2}}\right) 6^{\frac{1}{3}} / 3 \Gamma(1 / 3)$.

\subsection{Boundary Contributions}

We approximate the contour at $\phi=0$ by the straight line $\phi=i v$ with $v \in[0, \varepsilon]$ and obtain the dominant contribution at $\phi=0$,

$$
I^{b}(0) \sim-e^{N \rho(0)} \int_{0}^{\varepsilon} e^{-N\left|\rho^{\prime}(0)\right| v} v d v \sim-N^{-2} \frac{e^{N \rho(0)}}{\left|\rho^{\prime}(0)\right|^{2}}
$$

as $N \rightarrow \infty$. The approximation is the same near $\phi=2 \pi$. Therefore,

$$
I^{b}=I^{b}(0)+I^{b}(2 \pi) \sim-2 N^{-2} \frac{e^{N \rho(0)}}{\left|\rho^{\prime}(0)\right|^{2}}
$$

\subsection{Balance between Saddle and Boundary Contributions}

There are four possible balances from Section 2.1:

1) $\mathfrak{R} \mu \neq 0$ and $\sigma=\operatorname{sign} B(\mu) \neq 0,2) \mathfrak{R} \mu=0$ and $B(\mu)=0,3) \mathfrak{R} \mu<0$ and $B(\mu)=0$, 4) $\mathfrak{R} \mu=0$ and $B(\mu)<0$. 
In case 1), using $\ln z=\ln |z|+i \arg z+2 k \pi i, \quad k \in \mathbb{Z}$, the balance between (10) and (12) leads to

$$
\mu+\sigma \sqrt{1+\mu^{2}}+\ln \frac{1-\sigma \sqrt{1+\mu^{2}}}{\mu} \sim-\frac{3}{2} \frac{\ln N}{N}-\frac{1}{2 N} \ln \left|\frac{\pi}{2 \mu^{2} \sqrt{1+\mu^{2}}}\right|-i \frac{\frac{\pi}{2}-\frac{1}{2} \arg \left(-\sigma \sqrt{1+\mu^{2}}\right)+\arg \left(\frac{-i}{\mu}\right)+2 k \pi}{N}
$$

As $N \rightarrow \infty$, this implies the limit curve

$$
\Re\left\{\mu-\sqrt{1+\mu^{2}}+\ln \frac{1+\sqrt{1+\mu^{2}}}{\mu}\right\}=0
$$

In case (2), from Section 2.1.2, the balance leads to the limit curve $\mathfrak{R} \mu=0,|\Im \mu| \geq 1$, i.e. the interval $\pm[i, i \infty)$. The balances in cases (3) and (4) lead to inconsistent results. The limit curve (14) is in agreement with Dubiner's result ([9] Equation (8.5)). It can be divided into three parts: the interval $\pm[i, i \infty$ ) and a curve connecting $\pm i$ in the left half-plane. The number of eigenvalues distributed around each part is given by the number of intersections of Equation (14) and

$$
\mathfrak{I}\left\{\mu-\sqrt{1+\mu^{2}}+\ln \frac{1+\sqrt{1+\mu^{2}}}{\mu}\right\}=\frac{2 k \pi}{N}
$$

Around $\pm[i, i \infty)$ are distributed about $\left(\frac{1}{2}-\frac{1}{\pi}\right) \frac{N}{2}$ eigenvalues respectively. There are left about $\left(\frac{1}{2}+\frac{1}{\pi}\right) N$ eigenvalues distributed around the curve connecting $\pm i$ in the lefthalf-plane. Figure 1 shows the limit curve and eigenvalue approximations for Cheybshev polynomials. The numerical eigenvalues are computed using 128-bit or 34 decimal digits of precision. They distribute near the limit curve except a large negative real eigenvalue $\mu \sim O(N)$, which is addressed in the next section. The asymptotic results are accurate even for small $N$ 's.

From Equation (13) we derive that the slowest decaying eigenmode has wave number

$$
\mu \sim-\frac{3}{2} \frac{\ln N}{N}-\frac{1}{2 N}\left[\ln \frac{\pi}{2}-\frac{1}{2} \ln \left(3 \frac{\ln N}{N}\right)\right] \pm b i
$$

where

$$
b \sim 1-\left[\frac{1}{2 N}\left(\frac{13 \pi}{4}-\frac{\sigma+1}{2} \pi-\frac{27}{8} \frac{\ln N}{N}\right)+\frac{\sigma}{2}\left(\frac{3}{2} \frac{\ln N}{N}\right)^{\frac{3}{2}}\right] /\left(1+\frac{1}{6 \ln N}\right) .
$$

The eigenvalues are plotted in Figure 3. They demonstrate better approximations than Dubiner's results ([6] Equation (8.6)).

If the boundary condition $u_{N}(-1)=0$ is imposed instead, then $\Re \lambda>0$, and the limit curve is a reflection by the imaginary axis.

\subsection{The Large Negative Eigenvalue}

The largest wave number limits the time step size of the numerical approximation. When $|\lambda|>O(N)$, the saddle points $\phi_{*}^{(1)}=i \sinh ^{-1}\left(\frac{N}{-\lambda}\right)$ and $\phi_{*}^{(2)}=2 \pi+i \sinh ^{-1}\left(\frac{N}{-\lambda}\right)$ are too close to the boundaries $\phi_{0}=0,2 \pi$. Thus we have to take the integration path of $I_{1}$ from 0 up to $\phi_{*}^{(1)}$, then to $\pi+i \infty$, and that of $I_{2}$ from $2 \pi$ up to $\phi_{*}^{(2)}$, then to $\pi+i \infty$, going down passing through $\phi_{*}^{(-1)}$ to $\pi-i \infty$. Both $\phi_{*}^{(1)}$ and $\phi_{*}^{(2)}$ are dominant saddles and $\phi_{*}^{(-1)}$ is subdominant. The integral then becomes 


$$
I=\int_{0}^{\pi+i \infty}+\int_{2 \pi}^{\pi-i \infty}+e^{|\lambda|\left(\cos \phi+i \frac{N}{|\lambda|} \phi\right)} \sin \phi d \phi=I_{1}+I_{2}
$$

The balance between saddle and boundary contributions implies a polynomial equation for $x=N^{2} /|\lambda|$,

$$
\sum_{n=0}^{\infty} \frac{x^{n+1}}{n ! 2^{n}(2 n+1)}-\frac{x^{n}}{n ! 2^{n}}=0
$$

The real root of (18) approaches a constant as the degree of the equation increases. Solving the equation of degree 5 gives $|\lambda| / N^{2} \sim 0.5855$. This large negative eigenvalue is plotted in Figure 2. The approximation agrees well with numerical results.

\section{Jacobi Polynomials}

We now generalize the asymptotic analysis in Section 2 to Jacobipolynomials $P_{N}{ }^{(\alpha, \beta)}(\cos \phi)$ with weight function $w(\cos \phi)=(1-\cos \phi)^{\alpha}(1+\cos \phi)^{\beta}, \alpha, \beta>-1$.

\subsection{Approximation of Jacobi Polynomials}

We approximate Jacobi polynomials in two regions, i.e. near 0 and away from 0.

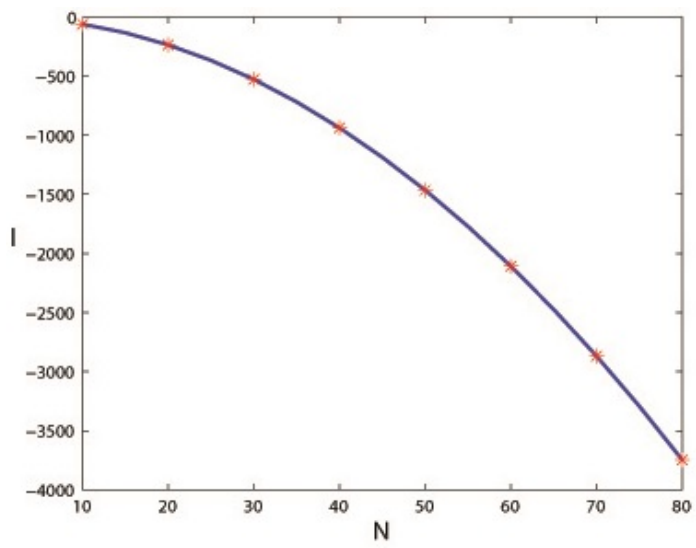

Figure 2. The large negative eigenvalue for Chebyshev polynomial, $\lambda \sim 0.5855 N^{2}$, confirmed by numerical approximations at $N=[10: 10: 80]$, indicated by *.
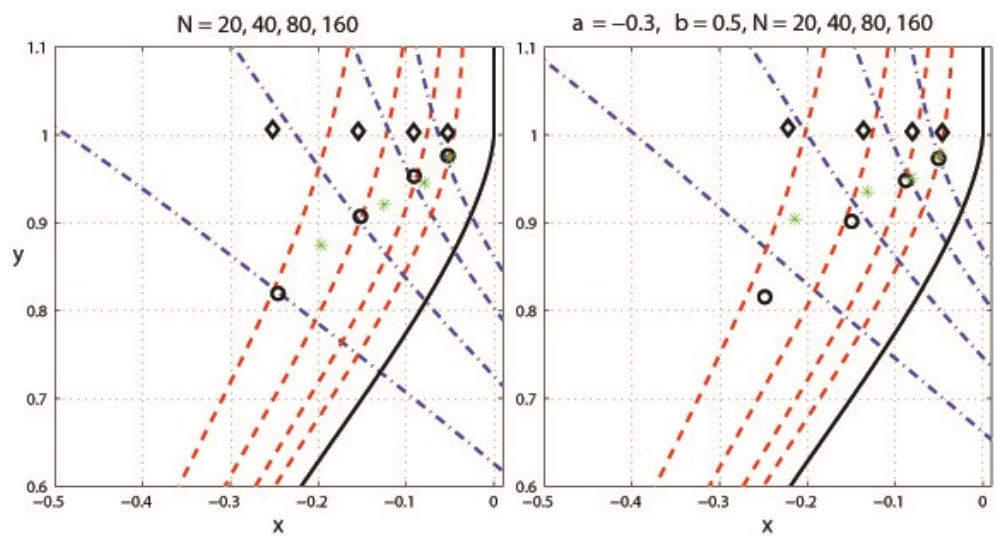

Figure 3. $\circ$ : the slowest decaying eigenmodes for Chebyshevpolynomial given by (16) (left) and Jacobi polynomialwith $\alpha=-0.3, \beta=0.5$ (right). $\diamond$ : Dubiner's approximation ([6] Equation (8.6)). *: numerical approximations. The limit curves move to the rightas $N$ increases. 
1) If $|\phi-n \pi| \geq \varepsilon$,

$$
P_{N}^{(\alpha, \beta)}(\cos \phi) \sim(2 \pi)^{-1 / 2}\left[e^{i N \phi} D\left(e^{-i \phi}\right)^{-1}+e^{-i N \phi} D\left(e^{i \phi}\right)^{-1}\right]
$$

where $D(z)=2^{-\frac{\alpha+\beta+1}{2}}(1-z)^{\alpha+\frac{1}{2}}(1+z)^{\beta+\frac{1}{2}}$, defined for $|z|<1,1<|z| \leq R<\infty, \lim _{|z| \rightarrow 1^{ \pm}} D(z)$ exists. We derived it from Szego [12] Theorem 12.1.2.

2) If $|\phi-0| \ll 1$,

$$
(\sin \phi)^{\frac{1}{2}+\alpha} P_{N}^{(\alpha, \beta)}(\cos \phi) \sim(2 \pi)^{-\frac{1}{2}} 2^{\frac{\alpha-\beta}{2}}\left[e^{i N \phi} e^{i \gamma}+e^{-i N \phi} e^{-i \gamma}\right]
$$

where $\gamma=-(\alpha+1 / 2) \pi / 2$. We derived it from Szego [12] Theorem 12.1.4.

\subsection{Limit Curve}

Using approximation (19), the integral in (4) becomes

$$
I=\int_{0}^{\pi+i \infty}+\int_{2 \pi}^{\pi-i \infty} e^{N \rho(\phi)} D\left(e^{-i \phi}\right)^{-1} \sin \phi d \phi,
$$

with the same $\rho(\phi)=-\mu \cos \phi+i \phi$. A similar analysis gives the saddle contribution

$$
I^{S} \sim N^{-1 / 2} e^{N \rho\left(\phi_{*}^{(\sigma)}\right)} e^{i \theta^{(\sigma)}} \frac{\sin \phi_{*}^{(\sigma)}}{D\left(e^{-i \phi_{*}^{(\sigma)}}\right)} \sqrt{\frac{2 \pi}{\left|\rho^{\prime \prime}\left(\phi_{*}^{(\sigma)}\right)\right|}}
$$

This is in agreement with the results for Chebyshev polynomials in Section 2, where

$$
D\left(e^{-i \phi}\right)=\left(i \sin \phi e^{-i \phi}\right)^{\alpha+1 / 2} \text { for } \alpha=\beta .
$$

Using the asymptotic formula for Bessel function of the first kind [13], $J_{\alpha}(z)=\left(\frac{2}{\pi z}\right)^{\frac{1}{2}} \cos (z+\gamma)+O\left(z^{-\frac{3}{2}}\right)$ as $|z| \rightarrow \infty$ with $\arg (z) \leq \pi-\delta, \delta>0$, we obtain

$$
J_{\alpha}\left(N \phi_{0}\right)=\left(\frac{2}{\pi N \phi_{0}}\right)^{\frac{1}{2}} \cos \left(N \phi_{0}+\gamma\right)+O\left(N^{-1}\right)
$$

As $N \rightarrow \infty$ for $\left|\phi_{0}-0\right| \ll N^{-1 / 3}$. From (20), we have

$$
P_{N}^{(\alpha, \beta)}(\cos \phi) \sim 2^{\frac{\alpha-\beta}{2}} N^{\alpha+\frac{1}{2}}(N \phi)^{-\alpha} J_{\alpha}(N \phi)
$$

Plug (23) to the integral in (4) and we obtain the boundary contribution

$$
I^{b}(0) \sim 0 \text { when } \alpha=0 .
$$

$$
I^{b}(0) \sim N^{\alpha-\frac{3}{2}} e^{-N \mu} 2^{\frac{\alpha-\beta}{2}}(2 \pi)^{\frac{1}{2}} \frac{2^{1-\alpha}}{\Gamma(\alpha)}
$$

\subsection{1. $\alpha \neq 0$}

The dominant balance is between saddle and boundary contributions. This gives the same limit curve as (14). The slowest decaying eigenmodes are plotted in Figure 3 for $\alpha=-0.3, \beta=0.5$.

The theorem in [9] proves that the eigenvalues lie in the left half-plane for Jacobi polynomial $P_{N}^{(\alpha, \beta)}$ if $-1<\alpha \leq 1$. We have derived asymptotically that the eigenvalue becomes unstable for $\alpha>1$.

3.2.2. $\alpha=0$

The dominant balance is between two saddle contributions from $\phi_{*}^{( \pm 1)}$, and this leads to a new limit curve

$$
\mathfrak{R}\left\{2 \sqrt{1+\mu^{2}}+\ln \frac{1-\sqrt{1+\mu^{2}}}{1+\sqrt{1+\mu^{2}}}\right\}=0
$$


The intervals $\pm[i, i \infty)$ are excluded because only one saddle point contributes.

The limit curve and eigenvalue approximations for Chebyshevpolynomials of the $2^{\text {nd }}$ kind ( $\left.\alpha=\beta=1 / 2\right)$ and Legendre polynomials $(\alpha=\beta=0)$ are plotted in Figures $\mathbf{4}$ and 5. The eigenvalues huddle around the limit curve. Note that even at a small $N=10$, the eigenvalue approximations for Legendre polynomials lie exactly on the dashed curve, which is true for all the other figures.
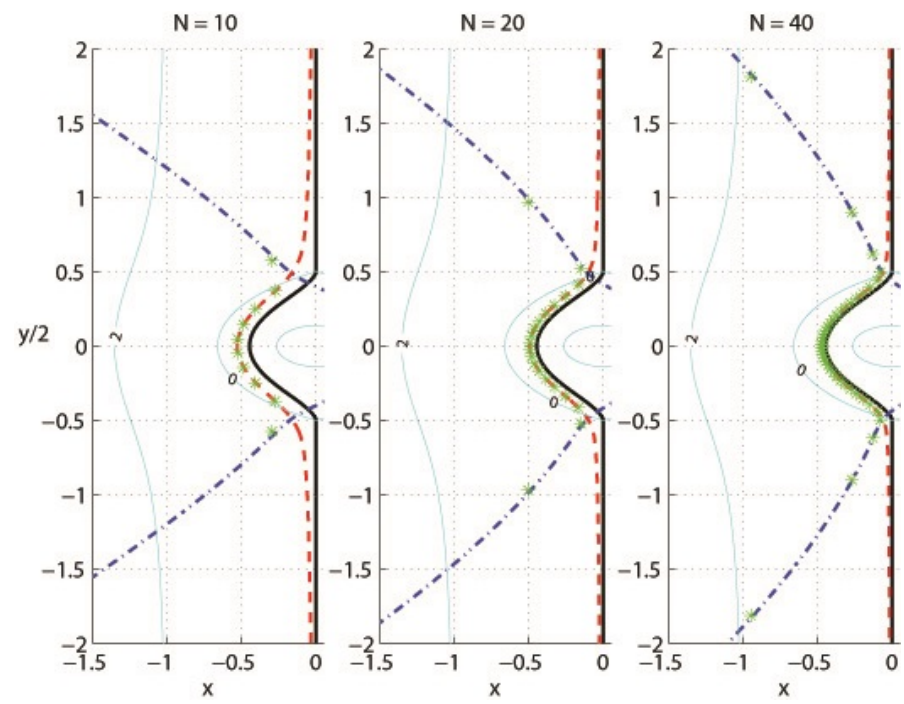

Figure 4. The limit curve and eigenvalue approximations for Chebyshev polynomials of the $2^{\text {nd }}$ kind $(\alpha=\beta=1 / 2)$. Thick solid:

$$
\begin{aligned}
& F(\mu, \sigma=-1)=0 \text {, dash: } F(\mu, \sigma=-1)=-\frac{1}{2} \frac{\ln N}{N}-O\left(\frac{1}{N}\right) \text {, dash-dot: } \\
& F(\mu, \sigma=1)=-\frac{1}{2} \frac{\ln N}{N}-O\left(\frac{1}{N}\right) .
\end{aligned}
$$
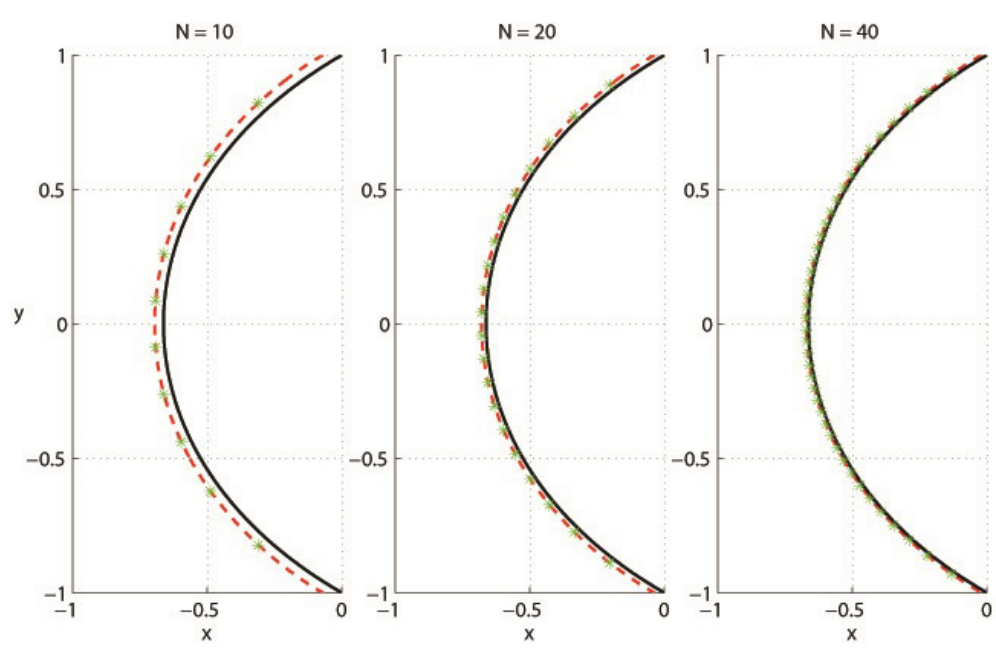

Figure 5. The limit curve and eigenvalue approximations for Legendre polynomials $(\alpha=\beta=0)$. Thick solid: $\mathfrak{R}\left\{2 \sqrt{1+\mu^{2}}+\ln \frac{1-\sqrt{1+\mu^{2}}}{1+\sqrt{1+\mu^{2}}}\right\}=0$, dash: $\Re\left\{2 \sqrt{1+\mu^{2}}+\ln \frac{1-\sqrt{1+\mu^{2}}}{1+\sqrt{1+\mu^{2}}}\right\}=\frac{1}{2 N} \ln \left|\frac{1+\sqrt{1+\mu^{2}}}{1-\sqrt{1+\mu^{2}}}\right|$. 


\subsection{Olver Paths}

When $\alpha=0$ and $|\mu \pm i| \ll 1$, the two saddle points $\phi_{*}^{(1)}=i \sin ^{-1}\left(-\frac{i}{\mu}\right)$ and $\phi_{*}^{(-1)}=\pi-i \sin ^{-1}\left(-\frac{i}{)}\right)$ nearly merge. They coincide at $\phi_{*}=\pi / 2$ when $\mu=-i$, and $3 \pi / 2$ when $\mu_{\mu}=i$. The expansions given $/$ by the ordinary method of steepest descents are not uniformly valid for $|\mu \pm i| \ll 1$. We need to construct uniform expansions near $\pm i$ and deform the contours intg Olver paths [14].

Apply the cubic transformation, $\rho(\phi, \mu)=\frac{1}{3} \zeta^{3}-\eta(\mu) \zeta+A(\mu)$, to map the saddle points of $\rho(\phi)$ in the $\phi$-plane to the saddle points $\pm \sqrt{\eta}$ in the $\zeta$-plane. It is shown [15] that the mapping is uniformly regular and one-to-one for all $\mu$ in a neighborhood of $\pm i$. The integral (21) becomes

$$
I=\int_{\mathcal{C}_{ \pm}} e^{N\left(\frac{1}{3} \zeta^{3}-\eta \zeta+A\right)} D\left(e^{-i \phi}\right)^{-1} \sin s \phi \frac{d \phi}{d \zeta} d \zeta
$$

where $\mathcal{C}_{ \pm}$is a contour running from $-\infty$ to $\infty e^{ \pm \pi i / 3}$ when $|\mu \pm i| \ll N^{-1}$, through the saddle point $\zeta=\sqrt{\eta}$. The first order approximation gives

$$
I^{S} \sim N^{-\frac{1}{3}} 2^{\frac{4}{3}} \pi e^{i N\left(\pi \mp \frac{\pi}{2}\right) \mp i \frac{5 \pi}{6}} \mathrm{Ai}\left(N^{\frac{2}{3}} 2^{\frac{1}{3}} e^{ \pm i \frac{\pi}{6}}(\mu \pm i)\right)
$$

for $|\mu \pm i| \ll N^{-1}$, where Ai is the Airy function. The eigenvalues are related to the zeros of the Bessel and Hankel functions of half-integer order $N+1 / 2$ [16]. Further discussions are presented in Section 4.

The slowest decaying eigenmode's wave number satisfies

$$
\operatorname{Ai}\left(N^{\frac{2}{3}} 2^{\frac{1}{3}} e^{ \pm i \frac{\pi}{6}}(\mu \pm i)+O\left(N^{-1}\right)\right)=0 .
$$

Ai (z) has a maximal zero $-c_{0}, c_{0}>0$ [17]. Then Equation (28) gives

$$
\lambda=N \mu \sim-N^{\frac{1}{3}} 2^{-\frac{1}{3}} e^{\mp i \frac{\pi}{6}} C_{0}+O\left(N^{-\frac{2}{3}}\right) \mp i[N+O(1)] .
$$

This is also the largest wave number (see Section 3.4). Hence when $\alpha=0$, the largest wave number is of order $N$ instead of $N^{2}$.

\subsection{The Large Negative Eigenvalue}

The formulas for saddle points contributions are not valid when the saddle points are too close to the boundaries at $|\lambda|>O(N)$. The approximation of Jacobi polynomials has to be replaced by Equation (20). If $\lambda$ is not real, we can take the same integration paths as in Section 3.2 and obtain the saddle contribution. The boundary contribution remains the same with $\lambda$ used first and then replaced by $N \mu$. The balance between saddle and boundary contributions when $\lambda \neq 0$, or between two saddle contributions when $\lambda=0$ gives the same limit curve as before.

If $\lambda$ is real negative, we take the integration paths as in Section 2.4. The balance leads to a polynomial equation for $x=N / \sqrt{|\lambda|}$,

$$
\sum_{n=0}^{\infty}\left(\begin{array}{c}
\frac{1}{2}-\alpha \\
n
\end{array}\right) c_{n} \cos \left(\alpha+\frac{n}{2}\right) \pi x^{1-n}+\left[\left(\frac{1}{2}-\alpha\right)\left(1-\sum_{n=0}^{\infty} \frac{x^{2 n}}{n ! 2^{n}}\right)+\sum_{n=0}^{\infty} \frac{x^{2 n+2}}{n ! 2^{n}(2 n+1)}\right] \sin \alpha \pi=0
$$

where $c_{n}=\sqrt{2 \pi} / 2(2 k-1)$ !! when $n$ is even $n=2 k ; c_{n}=2^{k} k$ when $n$ is odd $n=2 k+1$.

The lowest order approximation by solving Equation (30) of degree 2 gives

$$
x \sim \frac{-\sqrt{\pi / 2} \cot \alpha \pi+\sqrt{(\pi / 2) \cot ^{2} \alpha \pi+2(3 / 2+\alpha)(1 / 2-\alpha)}}{3 / 2+\alpha}
$$

For $-1<\alpha<0$. Figure 6 shows the ratio $\lambda / N^{2}$ obtained from (31) and by solving Equation (30) of degree 4, respectively, confirmed by the numerical eigenvalues at $N=40$ for different values of $\alpha$. The real root of the polynomial of degree $>2$ is negative for $0<\alpha<1 / 2$ and $1 / x$ approaches $-\infty$ as $\alpha$ approaches $1 / 2$ from the left. Thus, there is a large negative eigenvalue of $O\left(N^{2}\right)$ for $-1<\alpha<0$ and no large negative ei- 


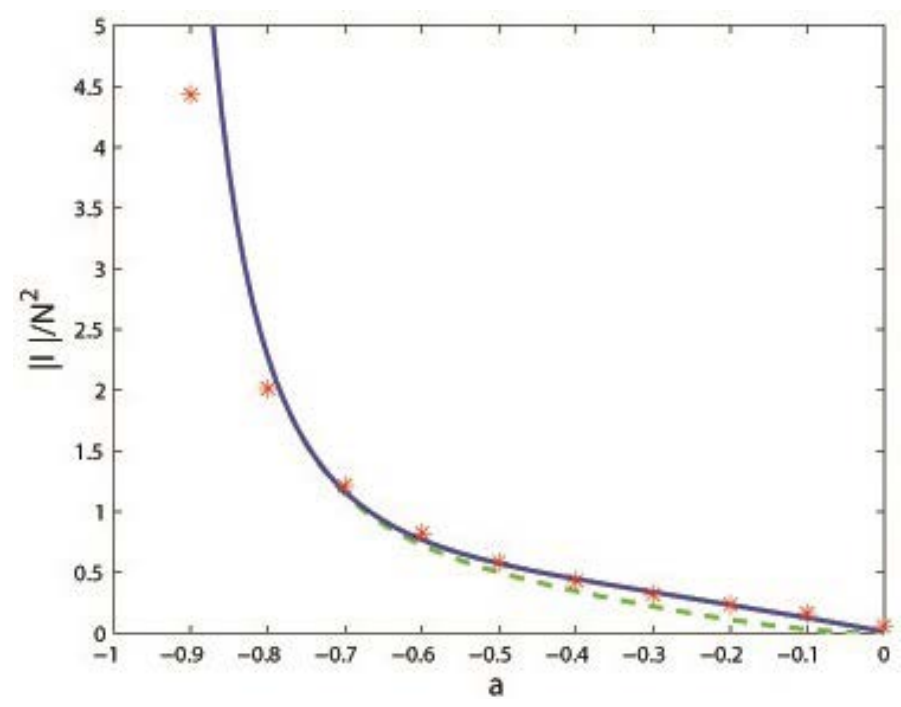

Figure 6. The large negative eigenvalue for $-1<\alpha<0$ scaled by $N^{2}$, compared with the numerical approximations for Jacobipolynomials at $N=40$ and $\alpha=[-0.001,-0.1:-0.1:-0.9]$, indicated as *. Solid: from solving Equation (30) of degree 4, dash: from Equation (31).

genvalue for $0<\alpha \leq 1 / 2$. The integral diverges for $\alpha>1 / 2$. Therefor there is a large negative eigenvalue of $O\left(N^{2}\right)$ for $-1<\alpha<0$.

When $|\alpha-0| \ll 1$, the assumption $|\lambda|>O(N)$ is no longer valid. In this case, the negative eigenvalue is simply described by the zero of the limit curve given in (14). When $\alpha=0$, the balance is between two saddle points. It gives same limit curve as (20). This is not consistent with the assumption $|\lambda|>O(N)$. So (29) also gives the largest magnitude eigenvalue which is $O(N)$.

\section{Legendre Polynomials and Spherical Bessel Functions}

Denote Legendre polynomials by $P_{N}=P_{N}^{(\alpha, \beta)}, \alpha=\beta=0$. As indicated in Section 3.3, the eigenvalues are directly related to the roots of spherical Bessel and Hankel functions.

The emission and scattering of electromagnetic radiation involves solving the vector wave equation [18]. The solutions are then expressed as an expansion in the orthogonal spherical waves, known as the multipole expansion. Consider the scalar wave equation $\Delta U-\frac{1}{c^{2}} \frac{\partial U}{\partial t}=0$, where $U=U(\mathbf{x}, t)$ is the velocity potential, $\mathbf{x}=(x, y, z)$ and $c$ is the speed of sound. For time-harmonic acoustic waves of the form $U(\mathbf{x}, t)=\mathfrak{R}\left\{u(\mathbf{x}) e^{-i \omega t}\right\}$ with frequency $\omega>0$, each Fourier harmonic $u(\mathbf{x})$ satisfies the Helmholtz equation $\Delta u+k^{2} u=0$ where $k=\omega / c$ is the wave number. It can be rewritten in terms of spherical coordinates $(r, \theta, \varphi)$. With the expansion $u=\sum f_{n}(r) Y_{n}(\theta, \varphi)$, the radial function $f_{n}(r)$ satisfies the equation

$$
r^{2} f_{n}{ }^{\prime}+2 r f_{n}{ }^{\prime}+\left[k^{2} r^{2}-n(n+1)\right] f_{n}=0
$$

known as the spherical Bessel differential equation [17] (10.1.1). With a change of variable $g_{n}(r)=\sqrt{r} f_{n}(r)$, $g_{n}$ satisfies the Bessel differential equation of half integer order $n+1 / 2$. Now let $r=i z / k$ and replace $f_{n}$ by $w, n$ by $N$, then Equation (32) becomes the modified spherical Bessel equation [17] (10.2.1)

$$
z^{2} w^{\prime \prime}+2 z w^{\prime}-\left[z^{2}+N(N+1)\right] w=0
$$

It has a solution, the modified spherical Bessel function of the third kind [17] (10.2.4), $k_{N}(z)=\sqrt{\frac{\pi}{2 z}} K_{N+\frac{1}{2}}(z)$, where $K_{N+\frac{1}{2}}(z)$ is the modified Bessel function of the third kind of order $N+1 / 2$. Using [17] (9.6.23) and integration by parts $N$ times, we obtain 


$$
k_{N}(z)=\int_{1}^{\infty} e^{-z s} P_{N}(s) d s=e^{-z} \int_{0}^{\infty} e^{-z t} P_{N}(1+t) d t
$$

Similar to deriving Equation (3),

$$
k_{N}(z)=\frac{e^{-z}}{z} \sum_{k=0}^{N} \frac{D^{k} P_{N}(1)}{z^{k}}
$$

Thus the eigenvalues $\lambda$ in (3) are the roots of $k_{N}(z)$, which are also the roots of Hankel function of order $N+1 / 2$, spherical and modified spherical Hankel functions using the relations [17] (10.1.1),(10.2.15).

\section{Collocation}

The Chebyshev collation method and stability analysis was discussed in [2], and then extended to general Gauss-Radau collocation methods [8] for the one-dimensional wave equation. The Gauss-Radau and GaussLobatto Jacobi methods were compared and it is shown that the latter is asymptotically stable for $\alpha \leq 1 \leq \beta$ [8]. In particular the only Gauss-Lobattoultraspherical method which is marginally stable corresponds to $\alpha=\beta=1$.

We take the discretization $-1=x_{0}<x_{1}<\cdots<x_{N}=1$ of the interval [-1,1], and approximate $u(x)$ by a polynomial $u_{N}(x)$ of degree $N$ that satisfies

$$
D u_{N}=\lambda u_{N}, \text { for } x=x_{1}, \cdots x_{N-1}, u_{N}(1)=0
$$

$u_{N}(x)$ interpolates the values $a_{0}, a_{1}, \cdots a_{N-1}, 0$ at $-1=x_{0}<x_{1}<\cdots<x_{N}=1$ and can be written as $u_{N}(x)=\sum_{J=0}^{N-1} a_{j} l_{j}(x)$, where $\left\{l_{j}(x)\right\}$ are the cardinal functions. Substituting $u_{N}$ into (33) gives the matrix system with eigenvalue $\lambda$. Collocation at zeros of orthogonal polynomials (Gauss points) is identical to the Tau method with Gauss quadrature. We consider three sets of Gauss-Lobattopoints $\mathbf{x}=\left[x_{1}, \cdots x_{N-1}\right]^{T}$ :

1) Chebyshev extreme points: $\left.x_{j}=-\cos \frac{j \pi}{N} ; 2\right)$ Chebyshev extreme points of the $2^{\text {nd }}$ kind: $x_{j}=j$ th zero of $\left.U_{N}{ }^{\prime} ; 3\right)$ Legendre extreme points: $x_{j}=j$ th zero of $P_{N}{ }^{\prime}$.

They can all be described as zeros of $Q_{N}{ }^{\prime}(x)$, where $Q_{N}$ is Chebyshev, Chebyshev of the $2^{\text {nd }}$ kind and Legendre polynomial of degree $N$, respectively. Defineresidual $R_{N}(x)=(\lambda-D) u_{N}(x)$. It is a polynomial of degree $N$ that vanishes at $x=-1$ and zeros of $Q_{N}{ }^{\prime}(x)$. Thus $R_{N}$ takes the form $R_{N}(x)=(x+1) Q_{N}{ }^{\prime}(x)$. In general, for Jacobi polynomial $P_{N}^{(\alpha, \beta)}$, from [19],

$$
R_{N}(x)=(x+1) \frac{d}{d x} P_{N}^{(\alpha, \beta)}=\frac{1}{2}(N+\alpha+\beta+1)(x+1) P_{N-1}^{(\alpha+1, \beta+1)}
$$

Using (19) and (20), we obtain approximations of $R_{N}(\cos \phi)$ for $\phi$ away from 0 and near 0 . The balance remains valid with

$$
D_{\text {col }}\left(e^{i \phi}\right)=\frac{2}{\left(1+e^{i \phi}\right)^{2}} D\left(e^{i \phi}\right), \alpha_{\text {col }}=\alpha+1, \beta_{\text {col }}=\beta+1
$$

However, for Legendre extreme points, the balance is now between saddle and boundary contributions since $\alpha_{\text {col }}=1$, and the limit curve is the same as the other two. Figure 7 shows the eigenvalues obtained using the Tau and collocation methods, together with their limit curves for Chebyshev ( $\alpha=\beta=-1 / 2$ ), Chebyshev of the $2^{\text {nd }}$ kind ( $\alpha=\beta=1 / 2$ ) and Legendre ( $\alpha=\beta=0$ ) polynomials. Collocations at Chebyshev and Legendre extreme points are stable. Collocation at Chebyshev extreme points of the $2^{\text {nd }}$ kind becomes unstable. In general, collocation at Gauss-Lobatto points is stable when $\alpha+1 \leq 1$, i.e. $\alpha \leq 0$, unstable when $\alpha>0$. There is no large negative eigenvalue with Chebyshev extreme points used. This can be derived asymptotically using the same method as before, or simply follows from $\alpha_{\text {col }}=\alpha+1$.

\section{Conclusions}

We have presented pseudospectral approximations of the first-order spectral differentiation matrices with zero 

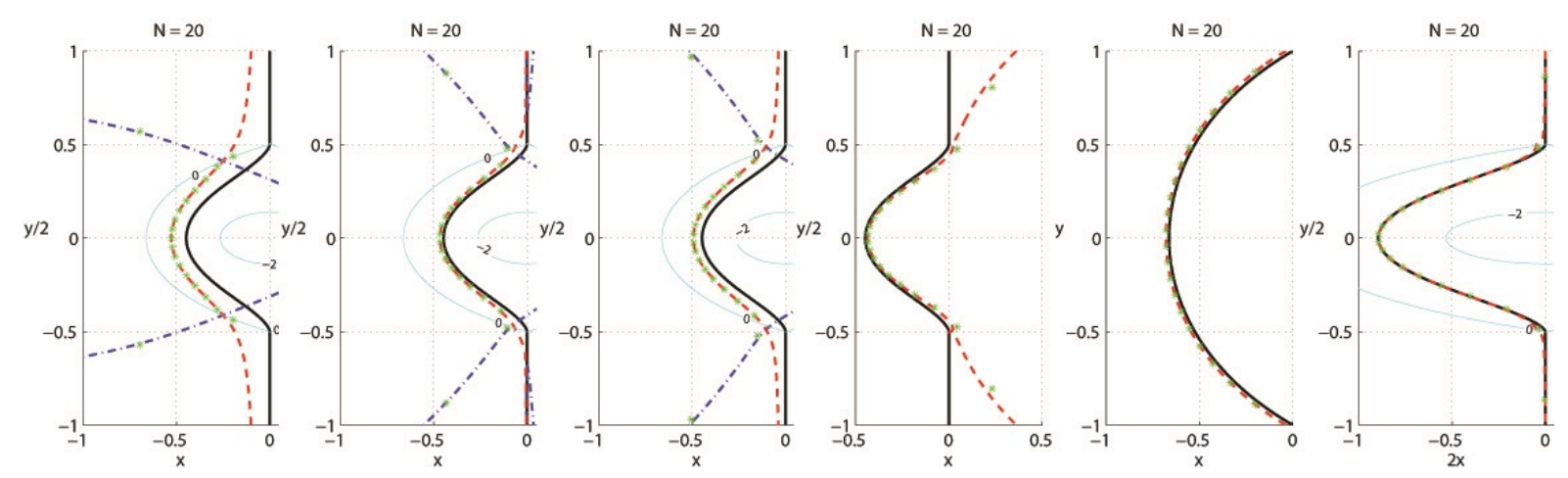

Figure 7. From left to right: Tau method at $\alpha=\beta=-1 / 2$, collocation at Chebyshev extreme points, Tau method at $\alpha=\beta=1 / 2$, collocation at Chebyshev extreme pointsof the $2^{\text {nd }}$ kind, Tau method at $\alpha=\beta=0$, collocation at Legendre extreme points.

boundary condition. We use the method of steepest descent to deform the integration path without changing the value of the integral, in order to obtain the dominant contributions from saddle and boundary points. This approach leads to the asymptotic formulas for the eigenvalues of Jacobi Tau method. Numerical examples with Chebyshev of the $1^{\text {st }}$ and $2^{\text {nd }}$ kind and Legendre polynomials are presented. They agree well with the asymptotic analysis, even at small sizes.

The approximations for the slowest decaying modes give more accuracy than those obtained in [6]. The largest wave numbers have raised interest in stabilities in pseudospectral approximations. We show that a large negative eigenvalue of order $N^{2}$ appears for $-1<\alpha<0$. The collocation methods are also examined. There are no large negative eigenvalues for collocations at Gauss-Lobatto points.

For Jacobi polynomials, the eigenvalues lie in the left half-plane if $-1<\alpha \leq 1$. We show that the theorem is sharp by obtaining asymptotic results that indicate unstable eigenvalues for $\alpha>1$. The eigenvalues for Legendre polynomials are related to the roots of Bessel and Hankel functions of half-integer order, spherical and modified spherical Bessel and Hankel functions. These results complete Dubiner's earlier analysis [6].

\section{References}

[1] Canuto, C., Hussaini, M.Y., Quarteroni, A. and Zang, T.A. (1988) Spectral Methods in Fluid Dynamics. Springer, New York. http://dx.doi.org/10.1007/978-3-642-84108-8

[2] Gottlieb, D. (1981) The Stability of Pseudospectral Chebyshev Methods. Mathematics of Computation, 36, $107-118$. http://dx.doi.org/10.1090/S0025-5718-1981-0595045-1

[3] Trefethen, L.N. and Embree, M. (2005) Spectra and Pseudospectra: The Behavior of Nonnormal Matrices and Operators. Princeton University Press.

[4] Trefethen, L.N. and Trummer, M.R. (1987) An Instability Phenomenon in Spectral Methods. SIAM Journal on Numerical Analysis, 24, 1008-1023. http://dx.doi.org/10.1137/0724066

[5] Weideman, J.A.C. and Trefethen, L.N. (1988) The Eigenvalues of Second-Order Spectral Differentiation Matrices. SIAM Journal on Numerical Analysis, 25, 1279-1298. http://dx.doi.org/10.1137/0725072

[6] Dubiner, M. (1987) Asymptotic Analysis of Spectral Methods. Journal of Scientific Computing, 2, 3-31. http://dx.doi.org/10.1007/BF01061510

[7] Tal-Ezer, H. (1986) Spectral Methods in Time for Hyperbolic Equations. SIAM Journal on Numerical Analysis, 23, 11-26. http://dx.doi.org/10.1137/0723002

[8] Jackiewicz, Z. and Welfert, B.D. (2003) Stability of Gauss-RadauPseudospectral Approximations of the One-Dimensional Wave Equation. Journal of Scientific Computing, 18, 287-313. http://dx.doi.org/10.1023/A:1021121008091

[9] Csordas, G., Charalambides, M. and Waleffe, F. (2005) A New Property of a Class of Jacobi Polynomials. Proceedings of the AMS, 133, 3351-3560.

[10] Weideman, J.A.C. and Reddy, S.C. (2000) A Matlab Differentiation MatrixSuite. ACM Transactions on Mathematical Software, 26, 465-519. http://dx.doi.org/10.1145/365723.365727

[11] Arfken, G.B. and Weber, H.J. (1995) Mathematical Methods for Physicists. Academic Press. 
[12] Szego, G. (1939) Orthogonal Polynomials. AMS Colloquium Publication, 23.

[13] Waston, G.N. (1995) A Treatise on the Theory of Bessel Functions. 2nd Edition, Cambridge University Press.

[14] Olver, F.W.J. (1970) Why Steepest Descents? SIAM Review, 12, 228-247. http://dx.doi.org/10.1137/1012044

[15] Chester, C., Friedman, B. and Ursell, F. (1957) An Extension of The Method of Steepest Descents. Proc. Cambridge Philos. Soc., 53, 599-611. http://dx.doi.org/10.1017/S0305004100032655

[16] Driver, K.A. and Temme, N.M. (1999) Zero and Pole Distribution of Diagonal Padé Approximants to the Exponential Function. Questiones Mathematicae, 22, 7-17. http://dx.doi.org/10.1080/16073606.1999.9632055

[17] Abramowitz, M. and Stegun, I.A. (Eds.) (1965) Handbook of Mathematical Functions: With Formulas, Graphs, and Mathematical Tables. Dover Publications.

[18] Colton, D. and Kress, R. (1998) Inverse Acoustic and Electromagnetic Scattering Theory. 2nd Edition, Springer. http://dx.doi.org/10.1007/978-3-662-03537-5

[19] Doha, E.H. (2002) On the Coefficients of Eifferentiated Expansions and Derivatives of Jacobi Polynomials. J. Phys. A: Math. Gen., 35, 3467-3478. http://dx.doi.org/10.1088/0305-4470/35/15/308 